\title{
Spiral-Based Phononic Plates: From Wave Beaming to Topological Insulators
}

\author{
André Foehr, ${ }^{1,2}$ Osama R. Bilal, ${ }^{2,3,{ }^{*}}$ Sebastian D. Huber, ${ }^{3}$ and Chiara Daraio ${ }^{2, \dagger}$ \\ ${ }^{1}$ Department of Mechanical and Process engineering, ETH Zurich, 8092 Zurich, Switzerland \\ ${ }^{2}$ Division of Engineering and Applied Science, California Institute of Technology, Pasadena, California 91125, USA \\ ${ }^{3}$ Institute for theoretical Physics, ETH Zurich, 8093 Zurich, Switzerland
}

(Received 21 November 2017; published 15 May 2018)

\begin{abstract}
Phononic crystals and metamaterials can sculpt elastic waves, controlling their dispersion using different mechanisms. These mechanisms are mostly Bragg scattering, local resonances, and inertial amplification, derived from ad hoc, often problem-specific geometries of the materials' building blocks. Here, we present a platform that ultilizes a lattice of spiraling unit cells to create phononic materials encompassing Bragg scattering, local resonances, and inertial amplification. We present two examples of phononic materials that can control waves with wavelengths much larger than the lattice's periodicity. (1) A wave beaming plate, which can beam waves at arbitrary angles, independent of the lattice vectors. We show that the beaming trajectory can be continuously tuned, by varying the driving frequency or the spirals' orientation. (2) A topological insulator plate, which derives its properties from a resonance-based Dirac cone below the Bragg limit of the structured lattice of spirals.
\end{abstract}

DOI: 10.1103/PhysRevLett.120.205501

Phononic crystals and metamaterials have been used to manipulate waves in a wide frequency spectrum: from heat propagation at very high frequencies, ultrasonic waves at high frequencies, and audible sound and earthquake excitations at low or very low frequencies [1]. They generally consist of two- or three-dimensional unit cells arranged in periodic arrays. The advantage of designing building blocks in fundamentally discrete materials-like phononic materials - is the ability to engineer their dispersion relation. A common method to control the propagation or reflection of waves in dispersive systems is the opening of frequency band gaps, where waves cannot penetrate the material bulk. Moreover, these frequency bands can have unconventional characteristics, such as directing radially emitted waves to propagate only along predefined line(s) (wave beaming) [2], or along the edges of a medium, without being susceptible to imperfections or backscattering (waves with topological protection) [3].

Generally, opening a band gap can be accomplished utilizing three different physical mechanisms: (i) Bragg scattering (BS), where a periodic medium can inhibit waves whose wavelength is on the order of the mediums' spatial periodicity (i.e., the Bragg limit) through destructive interferences $[4,5]$. This is usually achieved by having two materials within the unit cell or a single material with holes. (ii) Local resonances (LR), where wave propagation can be restricted using an inherent resonance in the unit cell, decoupling the unit cell size from the wavelength of the attenuated waves and thereby enabling subwavelength wave control [6]. This resonance-based mechanism does not require the lattice to be periodic [7]. (iii) Inertial amplification (IA), where a resonator is connected to the unit cell in multiple points. The band gap opens due to the amplification of the effective inertia of the resonator [8]. The resonators' connections are usually achieved with hinges and/or rigid rods. All these band gap opening mechanisms can be employed to engineer dispersion and manipulate elastic waves for a multitude of applications, such as focusing, beaming, and insulation [9].

The design approaches for inducing each of these band gap mechanisms are vastly different (from material layering and holes for BS, pillar coatings and heavy inclusions for LR, to hinged mechanisms for IA) [9]. Additionally, the design of specific band properties - such as doubly negative properties—adds further complexity [10]. The need for a systematic design methodology — able to generate a plethora of physical phenomena for a variety of demanding applications-is apparent. Here, we present a platform for realizing different phononic metamaterial physics based on Archimedean spirals. Through the use of simple variations of the spirals' geometrical parameters and symmetry, we show that it is possible to realize BS, LR, and IA, with no layering, coating, added masses, or hinges. We present two proof-of-concept realizations for applications: (1) a plate to beam vibrations and (2) a plate with topological properties. In the first example, we show that it is possible to beam elastic waves in different directions, which can be changed continuously and independently of the lattice geometry. The beaming angle and the beam's focusing width vary as a function of the driving frequency and spirals' orientation. In the second example, we present the first topological metamaterial featuring a resonance-induced Dirac cone below the Bragg limit of the structured material. The presented platforms can integrate elementwise, real-time 


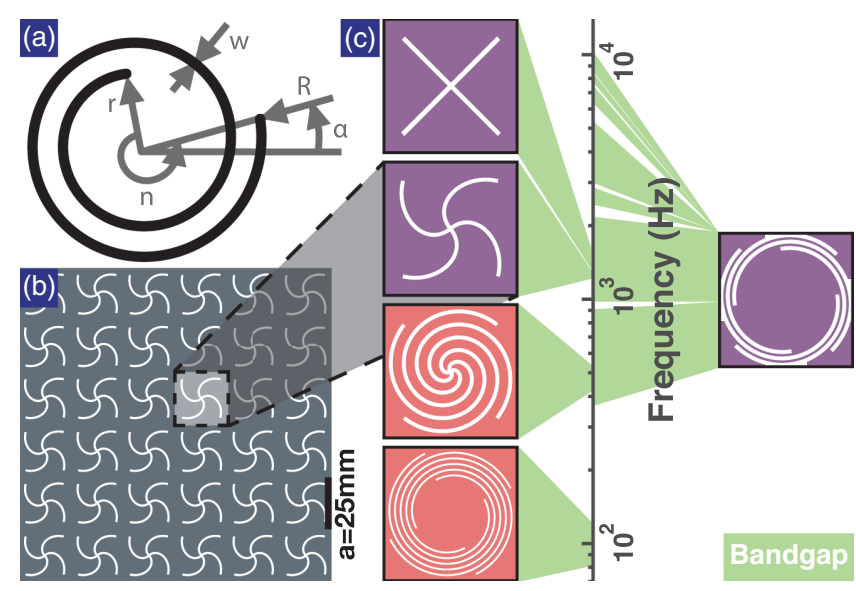

FIG. 1. Spiral-based structured plates. (a) An Archimedean spiral with its parameters. (b) Structured plate composed of a periodic array of spirals. White areas indicate void. (c) Variations of the spiral geometries with the corresponding flexural band gaps shaded in green. The band gaps span two decades of frequencies, while modifying the plate thickness or the lattice spacing. Unit cells exhibiting Bragg scattering are shown in purple and local resonances in red.

tunability [11] and can be easily produced by additive [11] or subtractive [12] manufacturing. In addition, the planar nature of the geometry is suitable for miniaturization, where optomechanical coupling is possible [13]. At the nanoscale, the subwavelength resonances can also have implications on the thermal conductivity of structured foils [14], where beaming or topological insulation for heat phonons is highly desirable.

Spirals are common in nature and art [15] and some of their mathematical bases have been known for millennia [16]. In phononics, spiral-based piezopatches enabled directional excitation and sensing [17,18]. Unispiral geometries embedded with heavy double-pillared inclusions have been used to show local resonance [19], and other chiral structures have already displayed negative refraction $[20,21]$ and wave beaming [22]. Recently, the phononic tunability of spiral-based metamaterials has been demonstrated $[11,12,23]$ and used to realize the first exclusively phononic transistor [12].

The polar representation of an Archimedean spiral [Fig. 1(a)] is $r(s)=R-(R-r) s, \phi(s)=2 \pi n s$, where $r$ is the inside radius, $R$ is the outside radius, $n$ is the number of turns, and $s \in[0 ; 1]$. Such spirals can be repeated periodically in any lattice configuration, producing a plethora of phononic properties. We start with a square lattice [Fig. 1(b)] with four concentric spirals $\left(\mathcal{C}_{4}\right.$ symmetry, with dispersion calculated along $\Gamma-X-M-\Gamma$ [24]) as voids within a solid plate. To characterize the dispersion properties of the different unit cells and identify the band gap locations, we model the material using the elastic wave equations for heterogeneous media [25] in an infinite lattice and apply Bloch boundary conditions [26]. We solve the
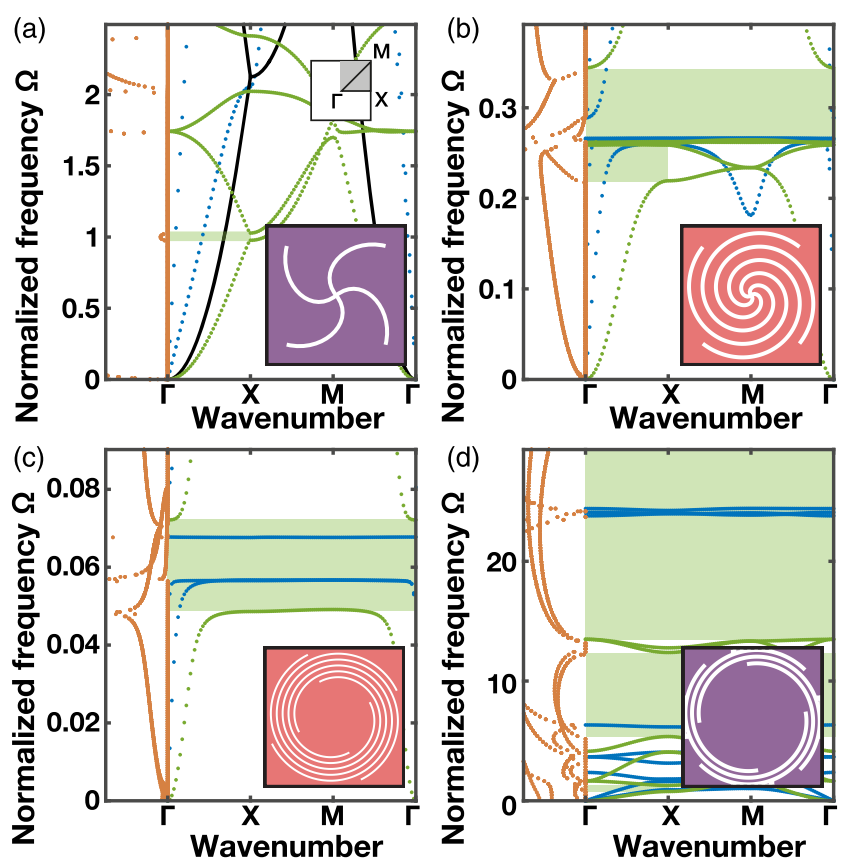

FIG. 2. Band gap opening mechanisms. Normalized dispersion curves with both the attenuated (orange) and propagating (blue for in-plane and green for out-of-plane) waves. The corresponding unit cells are in the inset. Band gaps are shaded in green. (a) Bragg scattering, (black line) flexural dispersion of the virgin material, (b) local resonance, (c) inertial amplification, and (d) Bragg scattering.

resulting equation using the finite element method (COMSOL). By varying the spirals' parameters, we open band gaps spanning more than 2 orders of magnitude in frequency for a constant plate thickness and lattice spacing [Fig. 1(c) and Video 1 in Supplemental Material [27]].

To identify the band gap opening mechanism and determine its Bragg scattering limit, we plot both propagative [i.e., real wave numbers [Figs. 2(a)-2(d), green and blue dots]] and attenuating [i.e., imaginary wave numbers [Figs. 2(a)-2(d), orange dots]] waves. While the Bragg limit in a given homogeneous medium has a fixed frequency per polarization, the introduction of other materials (or voids) within the unit cell alters this frequency significantly [Fig. 2(a), black lines versus green dots]. To center that frequency around 1 , we define $\Omega=f / f_{\text {Bragg }}$, where $f_{\text {Bragg }}$ is the computed Bragg limit for each unit cell (Fig. S1 of Supplemental Material [27]).

By incorporating a minimal spiral void, we induce a minute Bragg scattering partial band gap at $\Omega=1$ [Fig. 2(a), green shaded area] with a Bragg-like semicircular band within the attenuation profile [Fig. 2(a), orange dots]. The resulting unit cell is too stiff to support resonances; however, the impedance mismatch between the homogeneous material and the void acts as a periodic scatterer for elastic waves. By increasing the number of turns $(n)$, four curved beams emerge within the unit cell, 
acting as local resonators. This opens a subwavelength band gap, with inverted resonance peaks within the attenuation profile [Fig. 2(b)]. By increasing the inner radius of the spiral $(r)$, a single resonant mass emerges at the spiral core connected through four beams creating an inertially amplified unit cell [Fig. 2(c)]. This opens a deep subwavelength band gap with more pronounced inverted peaks within the attenuation profile [20,28]. By increasing the outer radius $(R)$, the spiral void intersects with the unit cell boundary. The system is similar to a diatomic springmass-lattice [29], explaining the opening of multiple Bragg scattering band gaps with semicircular attenuation profiles [Fig. 2(d)]. Surprisingly, (without normalization) the frequency of the first BS gap in [Fig. 2(d)] is similar to that of the LR geometry [Fig. 1(c)], reiterating the influence of geometry on the Bragg limit. We further investigate the effect of the parameters of the spiral pattern on its phononic band gaps (Fig. S3 of Supplemental Material [27]).

In addition to the geometric parameters of the individual spirals, the underlying unit cell symmetry plays a critical role in its phononic dispersion properties. An asymmetry within the unit cell, generating partial band gaps and pass bands, can lead to wave beaming with preferential paths for waves within a homogeneously structured media. Such irregular transmission of waves is commonly related to the static anisotropy of the material. In some cases, a change in beaming direction requires a complete redesign of the lattice $[2,22,30,31]$. In our platform, we demonstrate the ability to continuously tune the beaming direction without the need for redesigning the structure or changing of the lattice vectors.

To demonstrate frequency-dependent beaming, we utilize a unit cell created by two concentric spiral voids [Fig. 3(a), inset], resembling a resonator connected within the unit cell in two points (resembling an inertially amplified geometry). This design allows beaming to occur at subwavelength frequencies (i.e., with signal's wavelength larger than the spiral's outer diameters) (Figs. S2(a) and S2(b) in Supplemental Material [27]). IA not only decouples the operational frequency from the unit cell size but also relies on resonances that are only excited by a specific wave propagation direction. The design of the spirals' orientation with respect to the propagation direction controls the beaming trajectory (Fig. S4 [27]). The intrinsic chiral nature of the spirals enables dynamic tuning of the beaming direction varying the excitation frequency (Fig. S4 [27]). Furthermore, it is possible to vary the beaming direction controlling the spiral's resonance with an applied external field [11].

To identify the frequencies of interest we consider the first quadrant of the $\kappa_{x}$ and $\kappa_{y}$ space. The dispersion relation for flexural waves of the considered unit cell shows a deviation between the dispersion branches along the symmetry lines $\Gamma-X$ (blue) and $\Gamma-Y$ (orange) [Fig. 3(a)]. We harness this deviation, manifested in the same unit cell,

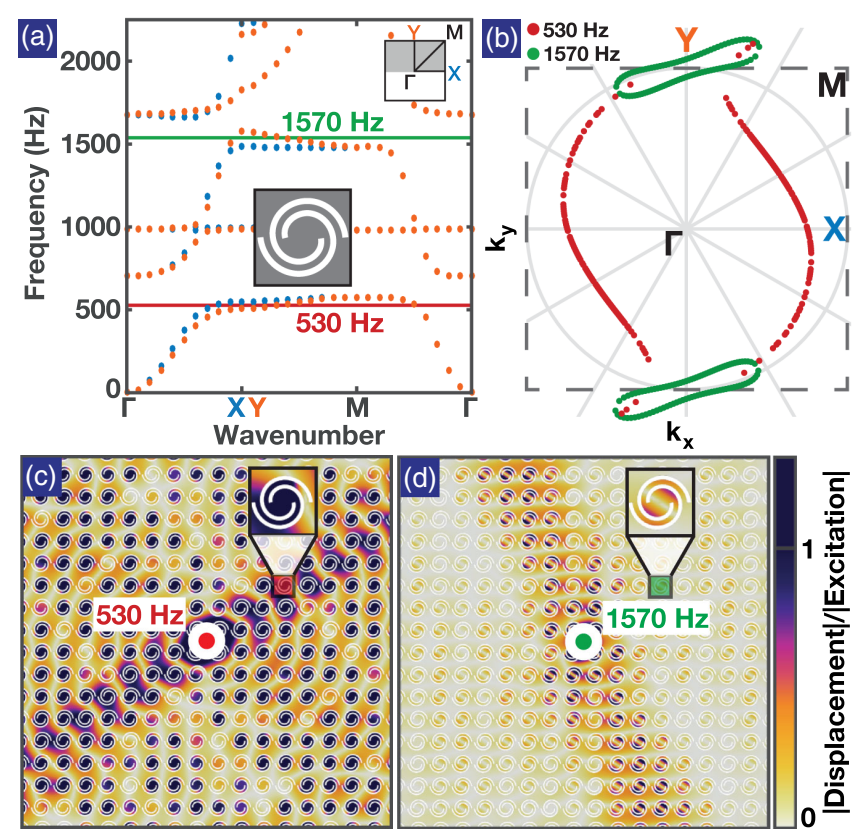

FIG. 3. Wave beaming. (a) Dispersion relation for elastic waves of the inset unit cell. (b) Isofrequency plot for the first Brillouin zone. The dashed gray lines indicate the unit cell edges in reciprocal space. (c) Frequency response of a finite plate, under out-of-plane harmonic excitation at $530 \mathrm{~Hz}$ and (d) $1570 \mathrm{~Hz}$ with absorbing boundary conditions.

to show wave beaming at two different frequencies: (i) in a pass band, at $f_{1}=530 \mathrm{~Hz}$ (red line), and (ii) in a stop band, at $f_{2}=1570 \mathrm{~Hz}$ (green line). A partial band gap exists at $f_{1}$ in the $\Gamma-Y$ direction, with a pass band at $f_{1}$ elsewhere. At $f_{2}$, a partial pass band exists in the $\Gamma-Y$ direction, with a band gap elsewhere. The projection of the eigensolutions on the full reciprocal space (i.e., the isofrequency contours) is plotted in Fig. 3(b). At $f_{1}$ (red) there exists no propagation along the $\Gamma-Y$ direction, with a strong preference for the waves to propagate along the diagonal of the first quadrant of the reciprocal space. At $f_{2}$ (green), the propagation is confined only along the $\Gamma-Y$ direction. The response of a finite sample with absorbing boundary conditions [Figs. 3(c) and 3(d)] - driven harmonically out of plane in the center, at $f_{1}$ and $f_{2}$ separatelyindicates a response similar to the infinite medium predictions. Within a pass band, at $f_{1}$, there exists a strong confinement of waves along a line, while waves still propagate radially in the plate. Within a band gap, at $f_{2}$, the wave propagation is also confined along a line; however, no propagation is allowed anywhere else in the plate.

The lattice vectors that govern the spacial packing of the spiral patterns can have a significant influence on BS band gaps. As for the band gaps resulting from resonances (LR and IA), periodicity is less important. However, some of the emerging phonon physics, such as phononic topological insulators [32-36], can benefit greatly from both periodicity 
and resonances resulting in subwavelength topologically protected band gaps. Topological insulators operating at subwavelength frequencies have been shown for both photonics and airborne acoustics [37,38]. Realizing such topological insulators for elastic phonons can redirect waves much larger than the unit cell size. At small scales, resonance-based topological insulators may have implications on resulting material thermal conductivity [14].

One avenue to design a subwavelength topological insulator is the opening of a band gap within a degenerate (duplicate) Dirac cone. First, we create a single Dirac cone for flexural waves, which is known to exist in a hexagonal packing. We utilize a hexagonal unit cell with six concentric spirals, resembling a resonator connected at six points to the unit cell ( $\mathcal{C}_{6}$ symmetry). This unit cell leads to a Dirac cone at $f=873 \mathrm{~Hz}$ [Fig. 4(a)] below the Bragg limit $(1661 \mathrm{~Hz})$ [Fig. S4(c) of Supplemental Material [27]]. We choose the unit cell thickness, $t_{h}=2 \mathrm{~mm}$, to ensure a full band gap for in-plane waves around $f$ [Fig. 4(a)]. In order to duplicate the Dirac cone, we consider a supercell (red) encompassing the original unit cell (blue) in addition to one-third of each of the neighboring unit cells [Fig. 4(b), left] [10,39]. Such an artificial enlargement of the unit cell boundaries results in doubling the branches within the dispersion curves through the folding of the cell's Brillouin zone [Fig. 4(b), right]. By modifying the spiral pattern of the original (blue) and the neighboring (red) unit cellsonly breaking its translational symmetry-the supercell becomes the smallest unit cell. The resulting unit cell manifests a topologically protected band gap at the $\Gamma$ point $[10,34]$, as a degenerate, yet inverted, mode exists at both edges of the gap [Fig. 4(c)]. By tiling two variations of the unit cell (as material I and II), a topological waveguide emerges at the interface [34]. To demonstrate the existence of a topological state at the interface between the two materials, we first calculate the dispersion curves of a quasifinite sample (periodic in one direction and finite along the other) composed of the two materials [Fig. 4(d)]. The two bands at the edge of the gap connect through two straight lines crossing at the $\Gamma$ point, with a mode shape showing clear localization of the wave at the interface [Fig. 4(d), right]. We simulate a plate composed of the two materials, in Fig. 4(e), to study the steady-state response of waves confined along the interface between the two materials (highlighted with a dashed black line) at $f$.

In summary, we show that the introduction of spiral patterns can greatly alter the dispersion characteristics of phononic media. By introducing these spiral patterns as voids within solid plates, we realize different phononic band gaps: Bragg scattering, local resonance, and inertial amplification. We capitalize on the internal unit cell symmetry, to create partial propagating bands within full band gaps and demonstrate two classes of subwavelength wave beaming, one within a propagating frequency and one within an attenuating one. The chirality of the structure

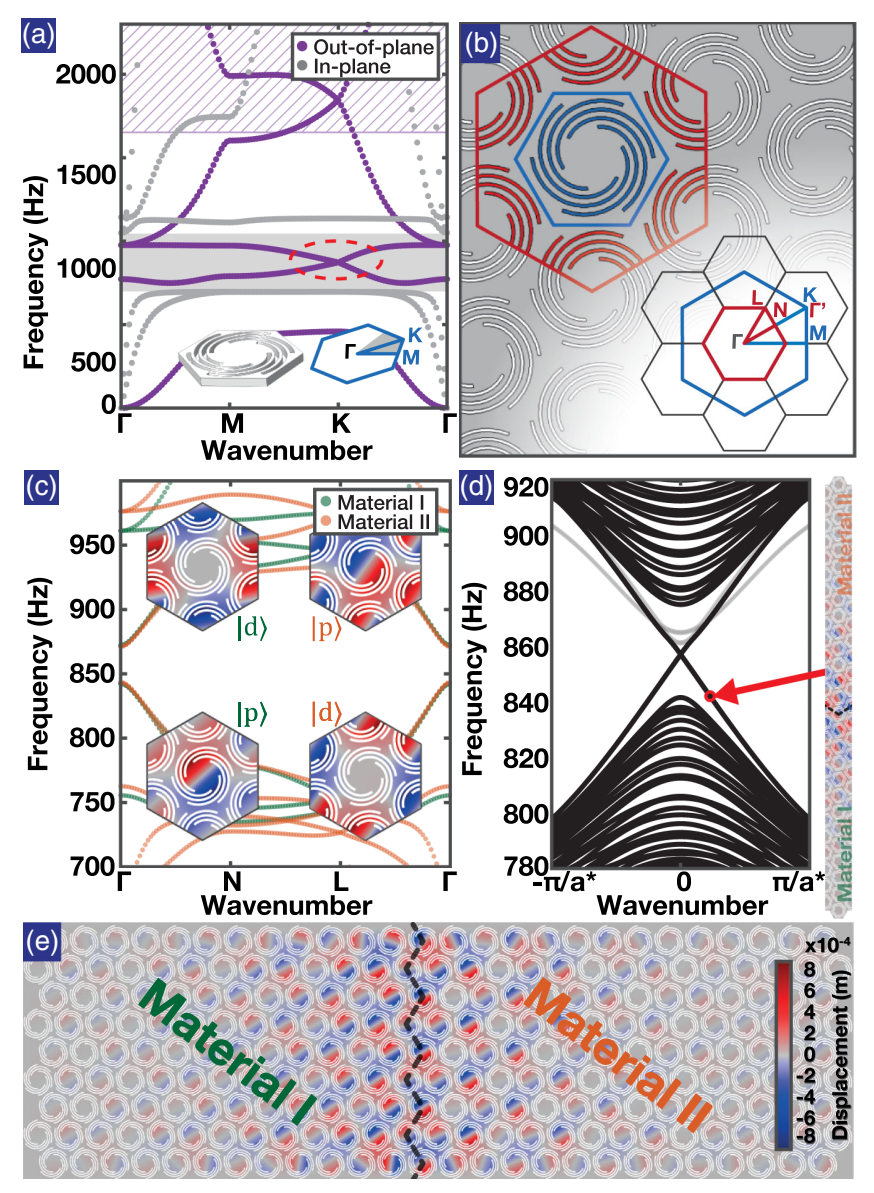

FIG. 4. Topological insulators. (a) Dispersion curves of the hexagonal unit cell in the inset with a flexural subwavelength Dirac cone highlighted in red and in-plane band gap highlighted in gray. Frequencies above the Bragg limit are hashed in purple. (b) Left: Supercell that includes the original hexagonal unit cell (blue) with additional neighboring spirals (red hexagon) in real space. Right: Folded symmetry line $(\Gamma-N-L-\Gamma)$ for the modified spiral patterns in reciprocal space. (c) Two dispersion curves of two supercells with small variations between the blue and red spirals (supercell I, $n_{\text {blue }}=1.02 n$ ) and (supercell II, $n_{\text {blue }}=1.03 n, w_{\text {blue }}=w_{\text {red }}=1.068 w$ ). The insets show the mode shapes of each unit cell at the edge of the topological gaps for super cells. (d) Dispersion curves for a quasifinite (periodic in one direction and finite with 27 spiral unit cells in the other) line of material I and II. In addition to trivial edge bands (gray), the previously observed band gap is closed by two topologically protected counterpropagating modes. The mode shape corresponding to the circled dispersion point (red), with the interface between the materials indicated by a black dashed line. (e) The mode shape at frequency $f=857 \mathrm{~Hz}$ for a finite sample composed of materials I and II.

appears to be at the origin of the frequency sensitivity of the beaming and could be of further use for other applications. Moreover, we alter the underlying lattice symmetry to create subwavelength-induced topologically protected band gaps. Our demonstrations show the potential of coupling various wave phenomena with a unified, 
easy-to-design and fabricate building blocks, enabling the design of novel phononic material plates. The presented advances highlight the versatility of spirals-based phononic systems.

The authors thank the reviewers for their helpful comments and A. Palermo for his support in the implementation of the complex eigenvalue computations. This work was supported by ETH Postdoctoral Fellowship FEL-26 15-2 to O. R. B. and ETH Grant No. ETH-24 15-2.

A. F. and O.R. B. contributed equally to this work.

*bilal@caltech.edu †daraio@caltech.edu

[1] M. Maldovan, Nature (London) 503, 209 (2013).

[2] R. Langley, J. Sound Vib. 197, 447 (1996).

[3] C. L. Kane and E. J. Mele, Phys. Rev. Lett. 95, 226801 (2005).

[4] M. Sigalas and E. N. Economou, Solid State Commun. 86, 141 (1993).

[5] M. S. Kushwaha, P. Halevi, L. Dobrzynski, and B. DjafariRouhani, Phys. Rev. Lett. 71, 2022 (1993).

[6] Z. Liu, X. Zhang, Y. Mao, Y. Zhu, Z. Yang, C. T. Chan, and P. Sheng, Science 289, 1734 (2000).

[7] M. Rupin, F. Lemoult, G. Lerosey, and P. Roux, Phys. Rev. Lett. 112, 234301 (2014).

[8] C. Yilmaz, G. M. Hulbert, and N. Kikuchi, Phys. Rev. B 76, 054309 (2007).

[9] M. I. Hussein, M. J. Leamy, and M. Ruzzene, Appl. Mech. Rev. 66, 040802 (2014).

[10] N. Kaina, F. Lemoult, M. Fink, and G. Lerosey, Nature (London) 525, 77 (2015).

[11] O. R. Bilal, A. Foehr, and C. Daraio, Adv. Mater. 29, 1700628 (2017).

[12] O. R. Bilal, A. Foehr, and C. Daraio, Proc. Natl. Acad. Sci. U.S.A. 114, 4603 (2017).

[13] T. Kan, A. Isozaki, N. Kanda, N. Nemoto, K. Konishi, M. Kuwata-Gonokami, K. Matsumoto, and I. Shimoyama, Appl. Phys. Lett. 102, 221906 (2013).

[14] B. L. Davis and M. I. Hussein, Phys. Rev. Lett. 112, 055505 (2014).

[15] T. A. Cook, Spirals in Nature and Art (E.P. Dutton, New York, 1903).

[16] T. L. Heath, The Works of Archimedes (Cambridge University Press, Cambridge, 1897).
[17] B. Yoo, A. Purekar, Y. Zhang, and D. Pines, Smart Mater. Struct. 19, 075017 (2010).

[18] E. Baravelli, M. Senesi, M. Ruzzene, L. De Marchi, and N. Speciale, IEEE Trans. Ultrason. Ferroelectr. Freq. Control 58, 7 (2011).

[19] S. Zhang, J. Hui Wu, and Z. Hu, J. Appl. Phys. 113, 163511 (2013).

[20] D. Bigoni, S. Guenneau, A. B. Movchan, and M. Brun, Phys. Rev. B 87, 174303 (2013).

[21] R. Zhu, X. Liu, G. Hu, C. Sun, and G. Huang, Nat. Commun. 5, 5510 (2014).

[22] A. Spadoni, M. Ruzzene, S. Gonella, and F. Scarpa, Wave Motion 46, 435 (2009).

[23] T. Jiang and Q. He, Appl. Phys. Lett. 110, 021907 (2017).

[24] F. Maurin, C. Claeys, E. Deckers, and W. Desmet, Int. J. Solids Struct. 135, 26 (2018).

[25] K. F. Graff, Wave Motion in Elastic Solids (Courier Corporation, North Chelmsford, 2012).

[26] F. Bloch, Z. Phys. A 52, 555 (1929).

[27] See Supplemental Material at http://link.aps.org/ supplemental/10.1103/PhysRevLett.120.205501 for additional information on the Bragg limit approximation, the bandgap evolution for spiral parameter variations and the influence of different spiral orientation angles on wave beaming.

[28] N. M. Frandsen, O. R. Bilal, J. S. Jensen, and M. I. Hussein, J. Appl. Phys. 119, 124902 (2016).

[29] L. Brillouin, Wave Propagation in Periodic Structures (McGraw-Hill Book Company, Inc. New York, 1946).

[30] M. Ruzzene and F. Scarpa, Phys. Status Solidi B 242, 665 (2005).

[31] A. S. Phani, J. Woodhouse, and N. Fleck, J. Acoust. Soc. Am. 119, 1995 (2006).

[32] R. Süsstrunk and S. D. Huber, Science 349, 47 (2015).

[33] S. H. Mousavi, A. B. Khanikaev, and Z. Wang, Nat. Commun. 6, 8682 (2015).

[34] C. Brendel, V. Peano, O. Painter, and F. Marquardt, Phys. Rev. B 97, 020102 (2018).

[35] R. K. Pal and M. Ruzzene, New J. Phys. 19, 025001 (2017).

[36] R. Chaunsali, C.-W. Chen, and J. Yang, Phys. Rev. B 97, 054307 (2018).

[37] S. Yves, R. Fleury, T. Berthelot, M. Fink, F. Lemoult, and G. Lerosey, Nat. Commun. 8, 16023 (2017).

[38] S. Yves, R. Fleury, F. Lemoult, M. Fink, and G. Lerosey, New J. Phys. 19, 075003 (2017).

[39] L.-H. Wu and X. Hu, Phys. Rev. Lett. 114, 223901 (2015). 